Nonlinear Processes in Geophysics, 12, 827-833, 2005

SRef-ID: $1607-7946 / \mathrm{npg} / 2005-12-827$

European Geosciences Union

(c) 2005 Author(s). This work is licensed

under a Creative Commons License.

\title{
Hysteresis-controlled instability waves in a scale-free driven current sheet model
}

\author{
V. M. Uritsky ${ }^{1}$ and A. J. Klimas ${ }^{2}$ \\ ${ }^{1}$ Institute of Physics, St. Petersburg State University, Russia \\ ${ }^{2}$ Code 692, NASA/Goddard Space Flight Center, Greenbelt, MD, USA
}

Received: 17 May 2005 - Revised: 12 July 2005 - Accepted: 12 July 2005 - Published: 20 September 2005

Part of Special Issue "Nonlinear and multiscale phenomena in space plasmas"

\begin{abstract}
Magnetospheric dynamics is a complex multiscale process whose statistical features can be successfully reproduced using high-dimensional numerical transport models exhibiting the phenomenon of self-organized criticality (SOC). Along this line of research, a 2-dimensional driven current sheet (DCS) model has recently been developed that incorporates an idealized current-driven instability with a resistive MHD plasma system (Klimas et al., 2004a, b). The dynamics of the DCS model is dominated by the scale-free diffusive energy transport characterized by a set of broadband power-law distribution functions similar to those governing the evolution of multiscale precipitation regions of energetic particles in the nighttime sector of aurora (Uritsky et al., 2002b). The scale-free DCS behavior is supported by localized current-driven instabilities that can communicate in an avalanche fashion over arbitrarily long distances thus producing current sheet waves (CSW). In this paper, we derive the analytical expression for CSW speed as a function of plasma parameters controlling local anomalous resistivity dynamics. The obtained relation indicates that the CSW propagation requires sufficiently high initial current densities, and predicts a deceleration of CSWs moving from inner plasma sheet regions toward its northern and southern boundaries. We also show that the shape of time-averaged current density profile in the DCS model is in agreement with steadystate spatial configuration of critical avalanching models as described by the singular diffusion theory of the SOC. Over shorter time scales, SOC dynamics is associated with rather complex spatial patterns and, in particular, can produce bifurcated current sheets often seen in multi-satellite observations.
\end{abstract}

Correspondence to: V. M. Uritsky

(uritsky@geo.phys.spbu.ru)

\section{Introduction}

Earth's magnetosphere is a driven spatially extended nonlinear system whose response to solar wind drive encompasses exceptionally broad ranges of temporal and energy scales. Statistical features of this response are described by robust power-law relations characteristic of critical self-organized avalanching systems (Consolini, 1997; Uritsky et al., 2002b, 2003). It has been suggested (Chapman et al., 1998; Uritsky and Pudovkin, 1998; Klimas et al., 2000) that the avalanching system of the magnetosphere resides in the magnetotail plasma sheet and is associated with collective behavior of multiple sporadic localized reconnections (Chang, 1999) which are known to appear during all phases of the magnetospheric substorm development (Angelopoulos et al., 1992; Sergeev et al., 1999; Nakamura et al., 2001; Sergeev, 2004).

In order to understand physical principles of scale-free plasma sheet behavior, a 2-dimensional driven current sheet (DCS) model has been developed that incorporates an idealized current-driven instability with a resistive MHD system (Klimas et al., 2004a, b). The DCS model is a continuum representation of gradient-controlled discrete transport models exhibiting the phenomenon of self-organized criticality (Bak et al., 1988; Lu, 1995; Klimas et al., 2000). An important characteristic of the DCM model is strong coupling between MHD and kinetic phenomena at respectively large and small scales of current sheet dynamics. This coupling involves a simplified mechanism of current-driven instability that, through its excitation and quenching, leads to the consequent growth and decay of the anomalous resistivity $\eta$ represented by the diffusion coefficient $D=c^{2} \eta / 4 \pi$. By analogy with discrete avalanche models, this idealized instability is assumed hysteretic so that its threshold for quenching is 
slightly below its threshold for excitation:

$$
\begin{aligned}
& Q(|J|)=\left\{\begin{array}{l}
D_{\min }, \quad|J|<\beta J_{c} \\
D_{\max },|J|>J_{c}
\end{array}\right. \\
& \frac{\partial D(x, z, t)}{\partial t}=\frac{Q(|J|)-D}{\tau}
\end{aligned}
$$

Here $J \equiv J_{y}$ is the $y$-component of the current density describing plasma sheet dynamics in the $x z$ plane, $J_{c}$ - critical current density, $\beta<1$ and $\tau$ - some constant parameters. At each spatial location, the quantity $Q$ can take one of two values, $D_{\max }$ or $D_{\min } \ll D_{\max }$, depending on $J$, and does not change within the interval $\left[\beta J_{c}, J_{c}\right]$.

The hysteretic behavior of the anomalous resistivity is expected to appear in various physical scenarios of currentdriven instability. In particular, it may accompany a generation of the electromagnetic wave field in the vicinity and above the ion gyrofrequency that can provide the anomalous resistivity necessary to initiate the magnetic reconnection (Yoon and Lui, 1996). As numerical simulations on the DCS model show, the hysteresis has several important consequences. At smallest spatial scales of the order of grid spacing, it influences nonlinear interactions of adjacent current sheet elements leading to their low-dimensional chaotic behavior characterized by fractional correlation dimension (Uritsky et al., 2002a). At largest scales, it produces global loading-unloading dynamics (Klimas et al., 2004a) reminiscent of the substorm cycle of Earth's magnetosphere. In the intermediate range of spatial scales, the DCS dynamics is dominated by scale-free diffusive energy transport characterized by a set of broadband power-law distribution functions (Klimas et al., 2004b) similar to those observed in the behavior of multiscale magnetospheric perturbations (Uritsky et al., 2002b).

The scale-free current sheet turbulence implies that individual current filaments can communicate over arbitrarily long distances within the available range of scales imposed by grid spacing and boundary conditions. The underlying mechanism of such communications manifesting themselves in current sheet waves (CSW) revealed numerically in DCS simulations (Klimas et al., 2004b) is strongly nonlinear. CSWs are active solitary waves accompanied by selfconsistent propagation of unstable current sheet regions in the form of moving spatially localized excitations. Until now, however, this intriguing phenomenon has not been investigated on a quantitative basis. The present paper is focused on this issue which can be of interest for both theoretical and experimental studies of plasma sheet dynamics. Based on a coarse-grained representation of the magnetic diffusion equation, we derive the expression for CSW speed as a function of hysteresis parameters. The derived relation indicates that under certain conditions, current-driven instabilities can propagate over considerable using the free energy accumulated in DCS. We demonstrate that such propagation requires sufficiently high initial current densities, and predict a deceleration of CSWs initiated in the vicinity of the neutral plane and moving toward northern and southern magnetotail lobes. We also show that the shape of time-averaged current density profile in the DCS model is consistent with steady-state spatial configuration of critical avalanching models as described by the singular diffusion theory of self-organized criticality. The results obtained are confirmed by numerical simulations and provide new clues for experimental exploration of multiscale plasma sheet dynamics.

\section{Results and discussion}

The standard MHD description of spatiotemporal evolution of the current density takes into account the diffusion of the magnetic field lines due to the anomalous resistivity mechanism as well as the plasma convection (Priest and Forbes, 2000):

$$
\frac{\partial \boldsymbol{J}}{\partial t}=\frac{c^{2}}{4 \pi}\left[\nabla^{2}(\eta(\boldsymbol{J}) \boldsymbol{J})-\nabla^{2} \frac{1}{c}(\boldsymbol{V} \times \boldsymbol{B})\right]
$$

It has been shown (Klimas et al., 2004b) that in order to reproduce the broadband scale-free turbulent plasma dynamics, the magnetic diffusion should be controlled by very thin current sheets created due to highly nonlinear dependence (Eq. 1) of the diffusion coefficient on local current values. In numerical simulations on the DCS model, the thickness of such transient current sheets appears to be of the order of grid spacing. Our main task will be to deduce the propagation speed of such current sheets as a function of plasma parameters.

According to the previous simulation results, the hysteresis-controlled current sheet waves propagate both in both $x$ and $z$ direction and are associated with spatial redistribution of $J_{y}$ component of the electric current. It has also been found that the CSW speed $v_{C S}$ mainly depends on the $z$ coordinate. On average, $v_{C S}$ gradually decreases as the excitations moves from the central plane $z=0$ toward upper and lower boundaries located at $z= \pm L_{z}$, and tend to stop at some small but nonzero distance from the boundaries. To describe these effects, consider a response of the DCS model to a localized perturbation described by the following initial conditions:

$$
\begin{aligned}
& D(x, z, t=0) \equiv D_{\min } \simeq 0, \\
& \left.\begin{array}{l}
J(x, z, t=0)>J_{c} \\
Q(x, z, t=0)=D_{\max }
\end{array}\right\} \quad z \in\left(z_{0}-\lambda / 2, z_{0}+\lambda / 2\right) \\
& \left.\begin{array}{l}
J(x, z, t=0)<\beta J_{c} \\
Q(x, z, t=0)=D_{\min }
\end{array}\right\} \quad-\text { in all other regions }
\end{aligned}
$$

Therefore, we assume that at time $t=0$, the values of the diffusion coefficient throughout the system are close to zero, and that the switching parameter $Q$ is also negligibly small everywhere except for a thin flat region centered at $z=z_{0}$ where the current density $J \equiv J_{y}$ has just exceeded the critical value $J_{c}$. By dividing the plasma volume into equally spaced thin layers parallel to the neutral DCS plane, and replacing continuous spatial derivatives with finite differences, Eq. (2) can be reduced to the coupled system of ordinary differential equations

$$
\frac{d J_{i}}{d t}=\left(D_{i-1} J_{i-1}+D_{i+1} J_{i+1}-2 D_{i} J_{i}\right) / \lambda^{2},
$$


$i=-L_{z} / \lambda, \ldots, L_{z} / \lambda$

in which $\lambda$ is the thickness of current sheet layers indexed by $i$. Since the instability excitation is a sporadic localized phenomenon predominantly controlled by the magnetic diffusion (Klimas et al., 2000), we omitted the convective term. Denote the active current layer $z \in\left(z_{0}-\lambda / 2, z_{0}+\lambda / 2\right)$ with $i=k$ and assign the value $J_{0}<\beta J_{c}$ to the initial current density in the adjacent $(k+1$-th) current layer. For simplicity, we shall also assume that the diffusion fluxes in $k-1$ th and $k$-th layers are linearly related through the condition $D_{k-1} J_{k-1} \approx \zeta D_{k} J_{k}$, with $\zeta \in(0,1)$ being a coupling constant. Using these assumptions, the system given by Eq. (4) can be rewritten for the chosen initial configuration as

$$
\left\{\begin{array}{l}
d J_{k} / d t=(\zeta-2) D_{k} J_{k} / \lambda^{2}, \\
d J_{k+1} / d t=D_{k} J_{k} / \lambda^{2}
\end{array}\right.
$$

The obtained equations provide a description of current density redistribution between active $\left(J>J_{c}\right)$ and quiet $\left(J<\beta J_{c}\right)$ current layers. During this interaction, the current density of the $k$-th layer gradually decreases making the current $J_{k+1}$ to increase (in the continuum limit, the opposite tendencies in $J_{k}$ and $J_{k+1}$ dynamics are due to the opposite signs of second spatial derivatives in these current layers).

Equations (5) are only valid until the time instant $t=t_{1}$ at which $J_{k+1}$ becomes greater than $J_{c}$. This limitation does not affect our calculation of the CSW speed since we are focused only on times $t \leq t_{1}$, which allows us to estimate $v_{C S}$ by the ratio $\lambda / t_{1}$ without analyzing the subsequent $J_{k+1}$ evolution. The solution to Eq. (5) depends on nonlinear dynamics of the diffusion coefficient $D_{k}$ which behaves differently before and after the time instant $t_{0}$ when the current $J_{k}$ drops below the $\beta J_{c}$ level. Assuming that the excitation transfer time $t_{1}$ is much smaller than the characteristic time $\tau$ of the diffusion coefficient relaxation, the evolution of $D_{k}$ as specified by Eq. (1) can be approximately described as

$D_{k}= \begin{cases}D_{\max }\left(1-e^{t / \tau}\right) \simeq \frac{D_{\max }}{\tau} t & t \leq t_{0}, \\ D\left(t_{0}\right) e^{-\left(t-t_{0}\right) / \tau} \simeq \frac{D_{\max }}{\tau} t_{0} & t>t_{0},\end{cases}$

Inserting Eq. (6) into Eq. (5) and applying the initial conditions specified above, the analytic expressions for time evolution of current densities in the interacting layers can easily be obtained:

$$
\begin{aligned}
& t \leq t_{0}:\left\{\begin{array}{l}
J_{k}=J_{c} e^{-(2-\zeta) \frac{D_{\max }}{2 \lambda^{2} \tau} \times t^{2}} \\
J_{k+1}=J_{0}+\frac{J_{c}}{2-\zeta}\left(1-e^{-(2-\zeta) \frac{D_{\max }}{2 \lambda^{2} \tau} \times t^{2}}\right),
\end{array}\right. \\
& t>t_{0}:\left\{\begin{array}{l}
J_{k}=\beta^{-1} J_{c} e^{-(2-\zeta) \frac{D_{\max }}{\lambda^{2} \tau} t_{0} \times t} \\
J_{k+1}=J_{0}+\frac{J_{c}}{2-\zeta}\left(1-\beta^{-1} e^{-(2-\zeta) \frac{D_{\max }}{\lambda^{2} \tau} t_{0} \times t}\right) .
\end{array}\right.
\end{aligned}
$$

Time $t_{0}$ entering the above relations is calculated from the condition $J_{k}\left(t=t_{0}\right)=\beta J_{c}$ which provides

$t_{0}=\lambda \sqrt{\frac{2 \ln (1 / \beta) \tau}{(2-\zeta) D_{\max }}}$,

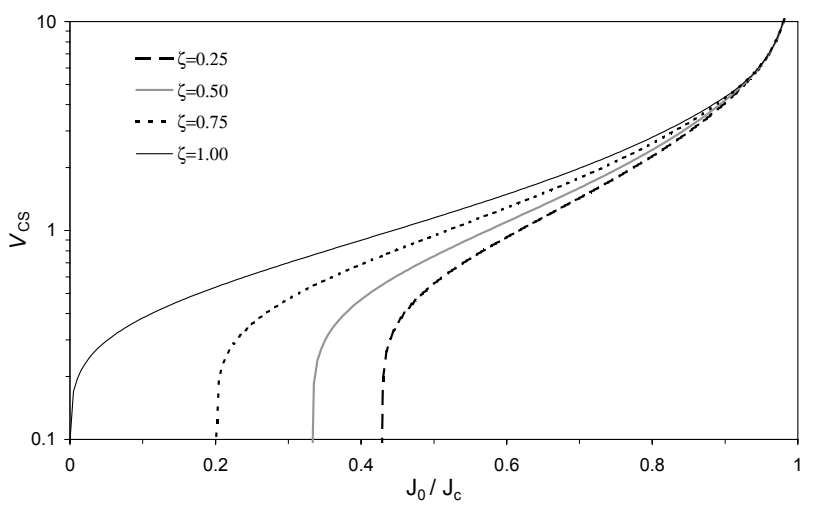

Fig. 1. Analytic dependence of the speed $v_{C S}$ of current sheet waves (CSW) on normalized initial current density plotted for different $\zeta$ values. In each case, CSW propagation condition is given by the inequality $J_{0} / J_{C}<(1-\zeta) /(2-\zeta)$.

The excitation transfer time $t_{1}$ required to switch the $k+1$ th current layer into the excited state with $Q=D_{\max }$ can be deduced from the condition

$J_{k+1}\left(t=t_{1}\right)=J_{c}$

In general case, this time may be less or greater than $t_{0}$. If $t_{1} \leq t_{0}$, the $k+1$-th layer switches to the unstable state while the diffusion coefficient $D_{k}$ continues to grow, and its dynamics is described by the first pair of the expressions given in Eq. (7). Otherwise, $D_{k}$ is roughly constant, and the second pair of expressions must be used. After the time $t_{1}$ has been estimated, the CSW speed is evaluated using the relation

$v_{a v l}=\frac{\lambda}{t_{1}\left(J_{0}, J_{c}, \beta, \tau, D_{\max }, \zeta\right)}$

giving a coarse-grained representation of $v_{C S}$ as a function of the initial current density, hysteresis parameters as well as the coupling constant $\zeta$. Using this approach, we have found that the CSW speed is given by the following formulas:

$v_{C S}= \begin{cases}\sqrt{\frac{(2-\zeta) D_{\max }}{2 \tau \ln \left(\frac{1}{1-(2-\zeta)\left(1-J_{0} / J_{C}\right)}\right)},} & 1-\frac{1-\beta}{2-\zeta} \leq \frac{J_{0}}{J_{C}}<1 \\ \frac{\sqrt{2(2-\zeta) D_{\max } \ln (1 / \beta) / \tau}}{\ln \left(\frac{1 / \beta}{1-(2-\zeta)\left(1-J_{0} / J_{C}\right)}\right)}, & \frac{1-\zeta}{2-\zeta} \leq \frac{J_{0}}{J_{c}}<1-\frac{1-\beta}{2-\zeta}\end{cases}$

These relations indicate that the wave speed goes down with the initial current density. As $J_{0}$ decreases, the expression under the logarithm sign in the denominator of the second branch of the solution (11) goes to zero, and the propagation speed becomes negligibly small. This condition corresponds to the limit

$J_{0} \rightarrow+J_{c}\left(\frac{1-\zeta}{2-\zeta}\right)$

By substituting different $\zeta$ values, we have built a family of $v_{C S}\left(J_{0}\right)$ dependences containing deceleration regions of different width (Fig. 1). In the extreme case $\zeta=1$ when the current layers with indexes $k-1$ and $k$ are strongly coupled, the 


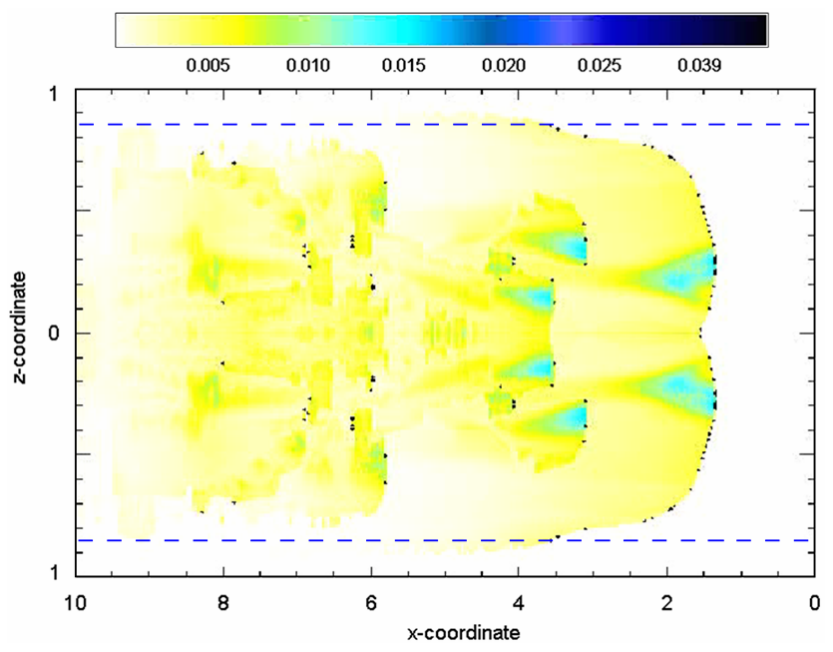

Fig. 2. A sample snapshot of anomalous resistivity distribution in the 2-D driven current sheet model (Klimas et al., 2004b). The SCW propagation region is limited by dashed horizontal lines corresponding to the propagation condition described by Eq. (12). Black dots are the unstable grid sites where the excitation condition $Q=D_{\max }$ is fulfilled.

CSW can propagate in regions with the arbitrarily low initial current density. In contrast, for very small $\zeta$, the deceleration region is fairly wide, and CSW can only penetrate into spatial domains where the current is sufficiently high $\left(J_{0} \geq 0.5 J_{c}\right)$.

To verify the obtained formulas, we have studied numerical runs of the 2-D driven current sheet model developed by (Klimas et al., 2004b). It has been found that usually, the coupling constant of the DCS model is close to the value $\zeta \approx 0.9$, which leads to the CSW propagation condition

$\frac{J_{0}}{J_{c}}>0.1$.

According to our simulation results, the spatial domain in which the instability waves can freely propagate does have a limited width in $z$ direction corresponding to the region where initial current densities exceed the level $0.1 J_{c}$ predicted by Eq. (13). Outside this region, the CSW mechanism of excitation transfer turns off, and current sheet dynamics is dominated by plasma convection. The CSW propagation region approximately coincides with the inner turbulent region of the current sheet where unstable sites with $Q>D_{\max }$ can exist, the effect clearly seen in our simulations (Fig. 2). We have also found that the transition between "fast" and "slow" solutions given by Eq. (11) should occur at $J_{0} / J_{c} \approx 0.9$. Such intense current densities are normally generated in the vicinity of the neutral plane.

The range of CSW speeds observed in simulations is also in a reasonable agreement with the theoretical result. To evaluate this range, we have investigated the motion of anomalous resistivity region boundaries using the double threshold condition

$S_{\text {diff }}(x, z, t)>a \wedge \partial S_{d i f f}(x, z, t) / \partial t>b$,

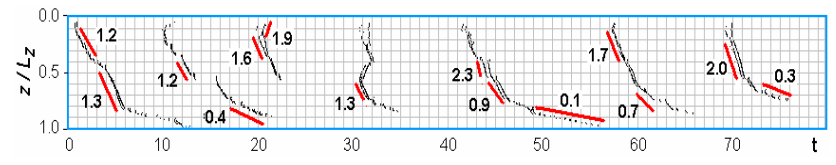

Fig. 3. Examples of spatiotemporal CSW propagation on the $z t$ plane $(x=2.5)$. Black dots show the grid sites satisfying Eq. (14) with the parameters $a=1.5 \times 10^{-4}, b=8.0 \times 10^{-4}$. Approximate estimates of speed values are given for each wave.

where $S_{\text {diff }}=|(c / 4 \pi) \eta \boldsymbol{J} \times \boldsymbol{B}|$ is the magnitude of the diffusive component of the Poynting flux, $a$ and $b$ are constants thresholds adjusted empirically to provide a reliable representation of CSW fronts. According to Eq. (14), we were looking for the places where the diffusive energy flux exceeded some constant value, and, at the same time, its time derivative was above some other value. Applying this criterion, spatiotemporal CSW traces have been visualized and a speed of about 200 waves has been estimated. It has been found that depending on CSW position and the local current density, $v_{C S}$ take values from the interval between 0 and 3.0 (Fig. 3). This range is consistent with the range of speeds calculated based on the relation (11) using $\zeta=0.9$ and varying $J_{0}$ parameter according to simulation results.

The solution (11) is not complete in the sense that it explicitly depends on the initial current $J_{0}$ which in its turn is defined by the prehistory of local magnetic field dynamics. In order to obtain the expression for average SCW speed at different spatial locations in a closed form, a time-averaged current density profile $\bar{J}(z)$ characterizing steady-state current sheet configuration has been chosen as a simplest substitute for $J_{0}$. Functional form of $\bar{J}(z)$ can be obtained using the results of the singular diffusion theory of self-organized critical avalanching models. It has been shown (Montakhab and Carlson, 1998; Carlson et al., 1990) that the long-term spatiotemporal evolution of such models can be described by the equations

$\frac{\partial \rho}{\partial t}=\nabla\left(D_{\text {sng }}(\rho) \nabla \rho\right), \quad D_{\text {sng }}(\rho)=\frac{1}{\left(\rho_{c}-\rho\right)^{\phi}}$,

in which $\rho$ is the relevant dynamical variable representing the quantity transported by the avalanche process, $D_{s n g}-$ the singular diffusion coefficient diverging as $\rho$ approaches the instability threshold $\rho_{c}, \phi-$ the order of the pole controlling the singularity of $D_{s n g}$ in the self-organized critical regime. In certain cases, the singularity order $\phi$ can be used as a free parameter allowing one to adjust the theory of singular diffusion to systems differing in local interaction rules, distribution of energy states and other local characteristics.

It has been found (Carlson et al., 1990) that onedimensional self-organized critical systems satisfying asymmetric boundary conditions

$\rho(1)=0, \quad D_{\text {sng }}(\rho(0)) \nabla \rho(0)=-f_{0}$ 


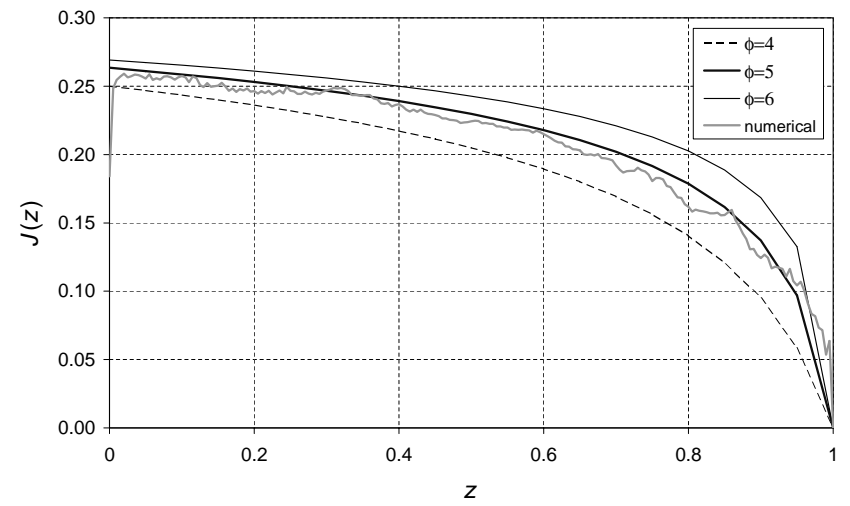

Fig. 4. Numerically estimated steady-state current density configuration in the 2-D current sheet model (averaging during one representative unloading interval) as compared to the singular diffusion solution presented by Eq. (21) with three different singularity orders $\phi$.

are characterized by the following universal asymptotic configuration at $t \rightarrow \infty$ :

$\rho(r, \phi>1)=\rho_{c}-\left[\rho_{c}^{-(\phi-1)}+f_{0}(\phi-1)(1-r)\right]^{-\frac{1}{\phi-1}}$

in which $r$ is the spatial coordinate. The conditions (16) correspond to open boundary at $r=1$ and closed boundary at $r=0$ where the fixed diffusive flux $f_{0}$ is applied. In case when the parameter $f_{0}$ is unknown, it can be estimated selfconsistently from the boundary value of the dynamical variable $\rho$ :

$f_{0}=\frac{\left[\rho_{c}-\rho(0)\right]^{-(\phi-1)}-\rho_{c}^{-(\phi-1)}}{\phi-1}$

The singular diffusion formalism has been successfully applied to various avalanching models, including cellularautomaton models of critical gradients (Carlson et al., 1990; Montakhab and Carlson, 1998) used as a discrete prototype for the DCS model (Lu, 1995; Klimas et al., 2000). In contrast to the nonlinear diffusion Eq. (1) describing a small-scale hysteretic evolution of anomalous resistivity, the solutions to Eq. (15) represent large-scale self-organization of spatial distribution of system's activity accompanying its evolution toward the critical state.

In the DCS model, the closest analogue to $\rho$ is the slowly varying current density configuration obtained by averaging over long intervals of time including many current driven instabilities. Let us show that the boundary conditions (16) satisfy the dependence of DCS current density on $z$ coordinate. First of these conditions is correct since the current density is always zero at $z=1$. The applicability of the second condition is not so obvious since the current is not fixed at $z=0$, and its time average appears to be a result of current sheet self-organization toward the stationary critical state in which the rate of the annihilation of oppositely directed magnetic fields along the neutral line is balanced by the rate at which the magnetic flux is pumped into the simulation box (Uritsky

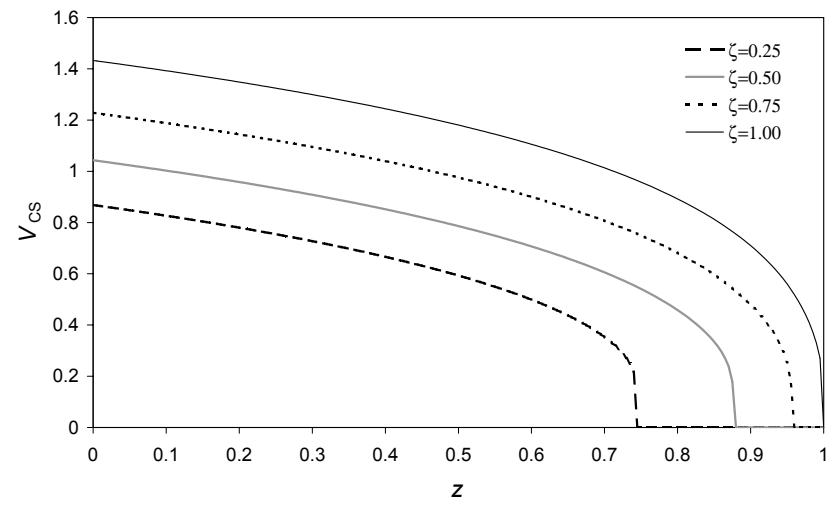

Fig. 5. Dependence of characteristic CSW speed on the $z$ coordinate at several different values of coupling parameter $\zeta$ plotted using the average current density profile described by Eq. (21) calculated based on the singular diffusion approach.

et al., 2002a). At small temporal scale of the order of the relaxation time of individual grid sites, current density variations along the line $z=0$ have a form of asymmetric pulses with rather steep left fronts evolving on the time scale $t_{0}$, and much flatter right fronts reflecting exponential relaxation of the diffusion coefficient with the characteristic time $\tau$. Neglecting the ascending fronts of the current density pulses and assuming that the mean frequency of pulses is of the order of $1 / \tau$, the average value of $J$ at the position $z=0$ can be approximately estimated as

$$
\langle J(z=0)\rangle_{t} \simeq \frac{1}{\tau} \int_{0}^{\tau} J_{c} e^{-t / \tau} d t=(1-1 / e) J_{c},
$$

which after substitution $\rho_{c} \equiv J_{c}$ leads to the following dependence of the boundary parameter $f_{0}$ on the singularity order:

$f_{0}=\frac{J_{c}^{-(\phi-1)}\left(e^{\phi-1}-1\right)}{\phi-1}$

Inserting the obtained formula into Eq. (17), we arrive at the asymptotic expression for steady-state current density distribution in the $z$ direction:

$\rho(r, \phi) \equiv \bar{J}(z, \phi)=J_{c}\left(1-\left[1+\left(e^{\phi-1}-1\right)(1-z)\right]^{-\frac{1}{\phi-1}}\right)$

By comparing the obtained solution with the results of numerical simulations on the DCS model, it has been found that Eq. (21) provides the best fit to average current density profiles at the singularity order $\phi=5$ (Fig. 4). Using this value and inserting Eq. (21) into Eq. (11), finally we obtain:

$v_{C S}(z) \simeq \frac{\sqrt{2(2-\zeta) D_{\max } \ln (1 / \beta) / \tau}}{\ln \left(\frac{1 / \beta}{1-(2-\zeta)(1+53.6(1-z))^{-1 / 4}}\right)}$.

For obtaining this expression, only the "slow" branch of the solution (11) has been used since the current density given by the relation (21) never exceeds the level 
$J_{c}(1-(1-\beta) /(2-\zeta))$ at which the "fast" solution comes into play. Since we have replaced the initial current $J_{0}$ with the time-averaged quantity $\bar{J}$, expression (22) can be considered as the estimation of characteristic CSW speeds at different $z$-locations (Fig. 5). The fact that actual (instantaneous) initial current densities may be essentially higher than $\bar{J}$ leads to underestimation of $v_{C S}$ values of certain waves. On the contrary, if the actual current density is below $\bar{J}$, the CSW speed appears to be below the characteristic value predicted by Eq. (22). As a result, some of the CSWs observed in numerical simulations (Fig. 3) propagate faster or slower than expected based on Eq. (22). On average, however, the obtained relation provides a reasonable approximation to spatial $v_{C S}$ dependence averaged over sufficiently long time intervals.

\section{Conclusions}

The propagating current sheets seem to represent a new type of cooperative plasma behavior which may play an important part in the dynamics of the magnetotail plasma sheet. Basic signatures of this type of behavior have been first described in (Klimas et al., 2000) and subsequently generalized to a more complete MHD system in (Klimas et al., 2004b). Our analysis has generated two groups of results that provide a more penetrating insight into this phenomenon in frames of the resistive MHD approximation:

1. Analytical relation for CSW speed as a function of plasma parameters controlling local anomalous resistivity dynamics has been derived that explains several effects discovered earlier in numerical simulations on the 2-D DCS model, such as the dependence of the CSW speed on the $y$-component of the electric current leading to wave deceleration in the vicinity of lower and upper current sheet boundaries, as well as the existence of spatial regions in which CSWs cannot propagate.

2. Asymptotic expression for global steady-state current density distribution has been found that characterizes average spatial configuration of the turbulent plasma sheet in the vicinity of self-organized critical state. Using this expression, the characteristic speed of current sheet waves as a function of distance from the central neutral plane has been estimated.

Physical mechanism of current sheet waves involves cooperative evolution of multiple current-driven instabilities at the spatial scale of kinetic processes underlying hysteretic response of the anomalous resistivity assumed in the DCS model. This scale defines rather thin current layers which can propagate over considerable distances and create conditions for scale-free evolution of anomalous resistivity regions and multiscale energy dissipation. Thin current sheet structures resembling MHD solutions with a plain singularity have received a good deal of attention during the last decade. Many theoretical models of thin current sheets have been developed showing an important role of such structures in magnetic energy storage and release during the substorm development (see e.g. Zelenyi et al., 2002, 2004; Sitnov et al., 2004 and references therein). In additional to these findings, our analysis strongly suggests that under certain conditions, thin current sheets can also move in a form of solitary self-supporting waves giving rise to complex scale-invariant spatial patterns of activity in vast portions of the magnetotail. Existence of such waves Earth's magnetosphere can, in principle, be verified using measurement results of multiprobe missions capable to resolve fine 3D features of plasma dynamics and presents a challenging task for future research.

Acknowledgements. The authors are grateful to Dimitris Vassiliadis for interesting and productive discussions. The work of V.Uritsky was partly supported by RFBR grant 04-05-64938 and Intergeophysica Russian research program.

Edited by: A. C. L. Chian

Reviewed by: two referees

\section{References}

Angelopoulos, V., Baumjohann, W., Kennel, C. F., Coroniti, F. V., Kivelson, M. G., Pellat, R., Walker, R. J., Luhr, H., and Paschmann, G.: Bursty Bulk Flows in the Inner Central Plasma Sheet, J. Geophys. Res. - Space Physics, 97, A4, 4027-4039, 1992.

Bak, P., Tang, C., and Wiesenfeld, K.: Self-Organized Criticality, Phys. Rev. A, 38, 1, 364-374, 1988.

Carlson, J. M., Chayes, J. T., Grannan, E. R., and Swindle, G. H.: Self-organized criticality and singular diffusion, Phys. Rev. Lett., 65, 20, 2547-2550, 1990.

Chang, T.: Self-organized criticality, multi-fractal spectra, sporadic localized reconnections and intermittent turbulence in the magnetotail, Physics of Plasmas, 6, 11, 4137-4145, 1999.

Chapman, S. C., Watkins, N. W., Dendy, R. O., Helander, P., and Rowlands, G.: A simple avalanche model as an analogue for magnetospheric activity, Geophys. Res. Lett., 25, 13, 23972400, 1998.

Consolini, G.: Sandpile cellular automata and magnetospheric dynamics, in: Cosmic Physics in the Year 2000, edited by: Aiello, S. E. A., SIF, Bologna, 123-126, 1997.

Klimas, A. J., Uritsky, V. M., and Baker, D. N.: Scale-free avalanching and loading-unloading cycle in a driven current-sheet model, in: 7th International Conference on Substorms (ICS-7), edited by: Pulkkinen, T., Finnish Meteorological Institute, Levi, Finland, 15-22, 2004a.

Klimas, A. J., Uritsky, V. M., Vassiliadis, D., and Baker, D. N.: Reconnection and Scale-Free Avalanching in a Driven Currentsheet Model, J. Geophys Res. - Space Physics, 109, A2, A02218, $1-14,2004 b$.

Klimas, A. J., Valdivia, J. A., Vassiliadis, D., Baker, D. N., Hesse, M., and Takalo, J.: Self-organized criticality in the substorm phenomenon and its relation to localized reconnection in the magnetospheric plasma sheet, J. Geophys. Res. - Space Physics, 105, A8, 18 765-18 780, 2000.

Lu, E. T.: Avalanches in Continuum Driven Dissipative Systems, Phys. Rev. Lett., 74, 13, 2511-2514, 1995. 
Montakhab, A. and Carlson, J. M.: Avalanches, transport, and local equilibrium in self-organized criticality, Phyical Review E, 58, 5, 5608-5619, 1998.

Nakamura, R., Baumjohann, W., Schodel, R., Brittnacher, M., Sergeev, V. A., Kubyshkina, M., Mukai, T., and Liou, K.: Earthward flow bursts, auroral streamers, and small expansions, J. Geophys. Res. - Space Physics, 106, A6, 10 791-10 802, 2001.

Priest, E. and Forbes, T.: Magnetic reconnection: MHD theory and applications, 590 pp., Cambridge University Press, Cambridge, 2000.

Sergeev, V. A.: Bursty bulk flows and their ionospheric footprints, in: Multiscale Processes in the Earth's Magnetosphere: from Interball to Cluster, edited by: Nemecek, Z., Kluwer Academic Publishers, Netherlands, 289-306, 2004.

Sergeev, V. A., Liou, K., Meng, C. I., Newell, P. T., Brittnacher, M., Parks, G., and Reeves, G. D.: Development of auroral streamers in association with localized impulsive injections to the inner magnetotail, Geophys. Res. Lett., 26, 3, 417-420, 1999.

Sitnov, M. I., Lui, A. T. Y., Guzdar, P. N., and Yoon, P. H.: Currentdriven instabilities in forced current sheets, J. Geophys. Res. Space Physics, 109, A03205, 1-15, doi:10.1029/2003JA010123, 2004.

Uritsky, V. M., Klimas, A. J., and Vassiliadis, D.: Multiscale dynamics and robust critical scaling in a continuum current sheet model, Phys. Rev. E, 65, 4, 046113-1-046113-5, 2002a.
Uritsky, V. M., Klimas, A. J., and Vassiliadis, D.: Evaluation of spreading critical exponents from the spatiotemporal evolution of emission regions in the nighttime aurora, Geophys. Res. Lett., 30, 15, 1813-1816, 2003.

Uritsky, V. M., Klimas, A. J., Vassiliadis, D., Chua, D., and Parks, G.: Scale-Free Statistics of Spatiotemporal Auroral Emissions as Depicted by POLAR UVI Images: Dynamic Magnetosphere is an Avalanching System, J. Geophys. Res. - Space Physics, 107, A12, 1426-1436, 2002b.

Uritsky, V. M. and Pudovkin, M. I.: Low frequency 1/f-like fluctuations of the AE-index as a possible manifestation of selforganized criticality in the magnetosphere, Ann. Geophys., 16, 12, 1580-1588, 1998,

\section{SRef-ID: 1432-0576/ag/1998-16-1580.}

Yoon, P. H. and Lui, A. T. Y.: Nonlocal ion Weibel instability in the geomagnetic tail, J. Geophys. Res. - Space Physics, 101, A3, 4899-4906, 1996.

Zelenyi, L. M., Delcourt, D. C., Malova, H. V., and Sharma, A. S.: "Aging" of the magnetotail thin current sheets, Geophys. Res. Lett., 29, 12, doi:10.1029/2001GL013789, 2002.

Zelenyi, L. M., Malova, H. V., Popov, V. Y., Delcourt, D. C., and Sharma, A. S.: Nonlinear equilibrium structure of thin current sheets: influence of electron pressure anisotropy, Nonlin. Processes Geophys., 11, 579-587, 2004,

SRef-ID: 1607-7946/npg/2004-11-579. 